\title{
The endomorphism rings of some abelian varieties II
}

\author{
By Shigefumi MORI
}

(Received March 16, 1976)

\section{§ 0. Introduction}

In our previous paper [3], we studied the endomorphism rings of abelian varieties and proved, in particular, that the Jacobian variety $J$ of the "generic" hyperelliptic curve of arbitrary genus (in every characteristic) has the property $\widetilde{\text { End }} J \cong Z$ (for an abelian variety $A$ defined over a field $k$, End $A$ denotes the ring of endomorphisms of $A$ which are defined over the algebraic closure $\bar{k}$ of $k$ ).

In this paper, for an arbitrary natural number $g$, we construct Jacobian varieties $J$ of dimension $g$ defined over $\boldsymbol{Q}$ such that $\widetilde{\text { End }} J \cong Z$.

Let us consider the curve defined over $\boldsymbol{Q}$ :

$$
C=\operatorname{Proj} Q[X, Y, Z] /(F(X, Y, Z)),
$$

where $F(X, Y, Z)=Y^{2}-X^{2 g+1} Z+b X Z^{2 g+1}+(p c / 4) Z^{2 g+2}, \operatorname{deg} X=\operatorname{deg} Z=1$, $\operatorname{deg} Y=g+1$, and $b, c, p$ are integers with the following properties:

$\alpha) \quad p$ is an odd prime number $(>1)$ such that some positive power of $(p-1) / 2$ belongs to $g Z$,

B) $b \bmod . p$ is a primitive root of $\boldsymbol{F}_{p}=\boldsymbol{Z} / p \boldsymbol{Z}$,

r) G.C.M. $\{b, c\}=$ G.C.M.M. $\{b, 2 g+1\}=$ G.C.M. $\{c, g\}=1$,

ס) $c \equiv-p \bmod .4$.

It is easy to show that $C$ is a non-singular hyperelliptic curve of genus $g$ with a $Q$-rational point $P=V_{+}(\{Y, Z\})$. The Jacobian variety $J(C)$ defined over $\boldsymbol{Q}$ of $C$ has the required property:

\section{THEOREM 1. End $J(C) \cong Z$.}

For example, $(b, c, p, g)=\left(-2,-1,5,2^{n}\right),\left(-2,1,7,3^{n}\right)(n \in N)$ enjoy the properties $(\alpha),(\beta),(\gamma)$ and $(\delta)$. In general, it is proved by Dirichlet's theorem on prime numbers in arithmetic progressions that there exist triples $(b, c, p)$ with the properties $(\alpha),(\beta),(\gamma)$ and $(\delta)$ for every $g$. Thus the required Jacobian varieties $J$ are obtained for every $g$. 


\section{§ 1. Proof of Theorem 1}

First, we need :

LEMMA 2. $J(C)$ has everywhere semi-stable reduction over $Z$ (for the definition, see [2, Exposé IX, Definition 3.4]).

Proof. By a change of coordinates $Y_{1}=Y-Z^{g+1} / 2$, we see that

$$
F(X, Y, Z)=G\left(X, Y_{1}, Z\right)=Y_{1}^{2}+Y_{1} Z^{g+1}-X^{2 g+1} Z+b X Z^{2 g+1}+\frac{p c+1}{4} Z^{2 g+2}
$$

belongs to $Z\left[X, Y_{1}, Z\right]$ by the properties $(\alpha)$ and $(\delta)$. It is obvious that $F$, and hence $G$, are irreducible polynomials of $Q\left[X, Y_{1}, Z\right]$. Since $G$ is primitive in $Z\left[X, Y_{1}, Z\right], G$ is a prime element of $Z\left[X, Y_{1}, Z\right]$. Thus $D=$ Proj $Z\left[X, Y_{1}, Z\right] /\left(G\left(X, Y_{1}, Z\right)\right)$ is irreducible and reduced, and $D_{Q} \cong C$. In particular $D$ is flat over $Z$. We claim that every geometric fiber of $D$ over $Z$ is irreducible and reduced, and has at most ordinary double points (i.e. nodes of multiplicity 2 ) as singularities, and hence $D$ is a stable curve over $Z$, [1, Definition (1.1)]. First, it is easy to check that $D$ is smooth over $Z$ at every point of $B=V_{+}\left(\left\{2, Y_{1}, Z\right\}\right)$, and that

$$
D-B \cong \operatorname{Spec} Z[1 / 2][U, V] /\left(V^{2}-H(U)\right),
$$

where $U$ (or, $V$ ) corresponds to $X / Z$ (or, $Y / Z^{g+1}+1 / 2$, resp.), and $H(U)=$ $V^{2}-F(U, V, 1) \in Z[1 / 2][U]$. Let $q(>2)$ be a prime number, and $K$ an overfield of $\boldsymbol{F}_{q}$ which is algebraically closed. Let $\bar{H}(U)\left(\in \boldsymbol{F}_{q}[U]\right)$ be $H(U) \bmod . q$, then $\bar{H}(U)$ has no triple factors in $K[U]$. Indeed, it follows immediately, from the properties $(\alpha),(\beta)$ and $(\gamma)$, that $\bar{H}, d \bar{H} / d U$ and $d^{2} \bar{H} / d U^{2}$ have no common zeros. Hence $(D-B)_{K} \cong \operatorname{Spec} K[U, V] /\left(V^{2}-\bar{H}(U)\right)$ is irreducible and reduced, and has at most ordinary double points as singularities (the irreducibility follows from $\operatorname{deg} \bar{H} \equiv \mathbf{m o d}$. 2). Thus $D$ is a stable curve over $Z$, and hence $D_{R_{s}}$ is also a stable curve over $R_{s}$, where $R_{s}$ denotes the strict henselization of $\boldsymbol{Z}_{(s)}$ ( $s$ is an arbitrary prime number). By [1, Theorem (2.4)], $J(C)$ has semi-stable reduction over $R_{s}$. Hence $J(C)$ has everywhere semi-stable reduction over $Z$, by [2, Exposé IX, (3.4.0)]. q.e.d.

It is proved by $\mathrm{K}$. A. Ribet [6, Corollary 1.4] that every endomorphism of an abelian variety $A$ over $\boldsymbol{Q}$ is defined over $\boldsymbol{Q}$ if $A$ has everywhere semistable reduction over $Z$. Hence $\widetilde{\operatorname{End}} J(C)=\operatorname{End}_{Q} J(C)$ by Lemma 2 , where $\operatorname{End}_{\boldsymbol{Q}} J(C)$ denotes the ring of endomorphisms defined over $\boldsymbol{Q}$ of $J(C)$. Since End $J(C)$ is a $Z$-algebra which is free of finite rank as a $Z$-module [4, $\S 19$, Corollary 1], Theorem 1 is reduced to the assertion: 


$$
\operatorname{rk} \operatorname{End}_{Q} J(C)=1 \text {, }
$$

where rk $\operatorname{End}_{Q} J(C)$ denotes the rank of $\operatorname{End}_{Q} J(C)$. Hence, by Proposition 3 below, it suffices to show: (1) $H(U)$ (given in the proof of Lemma 2) is irreducible in $Q[U]$, and (2) the Galois group $G$ of the minimal splitting field of $H(U)$ over $\boldsymbol{Q}$ acts doubly transitively on the set $\{\alpha \in \overline{\boldsymbol{Q}} \mid H(\alpha)=0\}$. (1) follows immediately from the consideration of the Newton polygon of

$$
2^{2 g+1} H(U / 2)=U^{2 g+1}-2^{2 g} b U-2^{2 g-1} p c
$$

with respect to the 2-adic valuation of $\boldsymbol{Q}$. As for (2), it suffices to show that $G$ (considered, canonically, as a subgroup of the symmetric group $\Im_{2 g+1}$ ) contains a cycle of length $2 g$. Since $H(U) \equiv U\left(U^{2 g}-b\right) \bmod . p$, the above assertion is reduced to the irreducibility of $U^{2 g}-b$ in $F_{p}[U]$ (cf. [7, §61]). On the other hand, by [5, Theorem 428], ${ }^{1)} U^{2 g}-b$ is irreducible in $\boldsymbol{F}_{p}[U]$ if (i) $X^{d} \equiv b$ mod. $p$ has no solution $X$ in $\boldsymbol{F}_{p}$ for every divisor $d(>1)$ of $2 g$, and (ii) $-4 X^{4} \equiv b$ mod. $p$ has no solution $X$ in $\boldsymbol{F}_{p}$ if $g$ is even. Now, (i) follows from the property $(\beta)$, and (ii) follows from the properties $(\alpha)$ and $(\beta)$.

q.e.d.

Proposition 3. Let $k$ be a field of characteristic $p \neq 2$, and let $f(X)$ be an irreducible polynomial of degree $2 g+1$ in $k[X]$ which is squarefree in $\bar{k}[X](\bar{k}$ is the algebraic closure of $k$ ). The Jacobian variety $J(V)$ defined over $k$ of the non-singular curve

$$
V=\operatorname{Proj} k[X, Y, Z] /\left(Y^{2}-f(X / Z) Z^{2 g+2}\right)
$$

( $\operatorname{deg} X=\operatorname{deg} Z=1$ and $\operatorname{deg} Y=g+1$ ) satisfies the condition:

rk $\operatorname{End}_{k} J(V) \leq \#\{$ prime divisors of $f(X) /(X-\theta)$ in $k(\theta)[X]\}$,

where $\theta(\in \bar{k})$ is a zero of $f(X)$.

Proof. The non-singularity of $V$ is easily checked, and we omit it. Let $\bar{V}$ be $V_{\bar{k}}$, $G$ the Galois group of $\bar{k}$ over $k$, and $\theta=\theta_{1}, \theta_{2}, \cdots, \theta_{2 g+1}$ the zeros $(\in \bar{k})$ of $f(X)$. Let $P_{0}, P_{1}, \cdots, P_{2 g+1}$ be the $\bar{k}$-rational points of $\bar{V}$ defined by $Y=Z=0, Y=X-\theta_{1} Z=0, \cdots, Y=X-\theta_{2 g+1} Z=0$, respectively. Since the group $J(\bar{V})(\bar{k})$ of $\bar{k}$-rational points of $J(\bar{V})$ is isomorphic to the group of divisor classes of degree 0 of $\bar{V}$, the group $J(\bar{V})_{2}$ of 2-section points of $J(\bar{V})(\bar{k})$ is expressed as:

$$
\varphi: \bigoplus_{i=1}^{2 g+1}(Z / 2 Z)\left(P_{i}-P_{0}\right) /(Z / 2 Z) \sum_{i=1}^{2 g+1}\left(P_{i}-P_{0}\right) \stackrel{\sim}{\longrightarrow} J(\bar{V})_{2} .
$$

1) This reference was pointed out by Professor T. Shioda. 
First the homomorphism $\varphi$ can be defined because $\operatorname{div}\left(Y / Z^{g+1}\right)=\sum_{i=1}^{2 g+1}\left(P_{i}-P_{0}\right)$ and $\operatorname{div}\left(X / Z-\theta_{i}\right)=2\left(P_{i}-P_{0}\right)(i=1, \cdots, 2 g+1){ }^{2)}$ If $\operatorname{Ker} \varphi \neq 0$, then, after renumbering $\theta_{i}$ 's, we may assume that $\operatorname{Ker} \varphi$ contains $\sum_{i=1}^{j}\left(P_{i}-P_{0}\right) \bmod .2$ for some $j$ such that $1 \leq j \leq 2 g$. Thus $\sum_{i=1}^{j} P_{i}+(2 g-2-j) P_{0}$ is a member of $\left|\mathcal{O}\left((2 g-2) P_{0}\right)\right|$. Since $\left|\mathcal{O}\left(2 P_{0}\right)\right|$ is a pencil of degree 2 on the hyperelliptic curve $\bar{V}, \mathcal{O}\left((2 g-2) P_{0}\right)$ is the canonical bundle $K_{\bar{V}}$ of $\bar{V}$ and $\left|K_{\bar{V}}\right|$ is composite with $\left|\mathcal{O}\left(2 P_{0}\right)\right|$. This is a contradiction because $P_{1}+P_{n} \notin\left|\mathcal{O}\left(2 P_{0}\right)\right|$ if $n \neq 1$. Hence, it follows that $\varphi$ is an injection. Since $J(\bar{V})_{2}$ is of order $2^{2 g}, \varphi$ is necessarily an isomorphism. Next, let

$$
\psi:(Z / 2 Z) \otimes \operatorname{End}_{k} J(V) \rightarrow J(\bar{V})_{2}
$$

be defined by $\psi(f \bmod .2)=f\left(P_{1}-P_{0}\right)\left(f \in \operatorname{End}_{k} J(V)\right) . \quad \psi$ is an injection because $\left\{\left(P_{1}-P_{0}\right)^{g} \mid g \in G\right\}$ generates $J(\bar{V})_{2}$. Since $\psi$ is compatible with the action of $H=\left\{g \in G \mid \theta^{g}=\theta\right\}, \operatorname{Im} \psi$ is contained in the set $J(\bar{V})_{2}{ }^{H}$ of $H$-invariant elements of $J(\bar{V})_{2}$. Hence Lemma 3 is reduced to the assertion :

$$
\operatorname{dim}_{Z / 2 Z} J(\bar{V})_{2}{ }^{H}=\text { the number of } H \text {-orbits of }\left\{P_{2}, \cdots, P_{2 g+1}\right\},
$$

which is obvious by (3.1).

q.e.d.

Before closing this paper, we give an example in characteristic $p>0$. Let $\boldsymbol{F}_{p}(t)$ be a purely transcendental extension of $\boldsymbol{F}_{p}$, and let $J$ be the Jacobian variety over $\boldsymbol{F}_{p}(t)$ constructed as in Lemma 3 from

$$
f(X)=X^{2 g+1}+X+t \in F_{p}[X] .
$$

Then :

THEOREM 4. End $J \cong Z$ if $p \nmid 2 g(2 g+1)$.

Proof. By a direct calculation, it is proved that

(4.1) $J_{k}$ has everywhere semi-stable reduction over $P_{\vec{F}_{p}}^{1}$ if $p \nmid 2 g(2 g+1)$, where $\boldsymbol{P}_{\bar{F}_{p}}$ has the function field $k=\overline{\boldsymbol{F}}_{p}\left(t^{1 / 2(2 g+1)}\right)$,

(4.2) $J$ has semistable reduction oyer $\overline{\boldsymbol{F}}_{p}[t]_{(t)}$ if $p \nmid 2 g$.

Let $k^{\prime}$ be the field of definition for an endomorphism $\varphi \in$ End $J_{\bar{F}_{p}(t)}$. Since $k\left(k^{\prime}\right)$ is a finite unramified extension field of $k$ by (4.1) [6, Theorem 1.3], it follows that $\overline{\boldsymbol{F}}_{p}(t) \subseteq k^{\prime} \subseteq k$. Similarly, by (4.2), $k^{\prime}$ is unramified over $\overline{\boldsymbol{F}}_{p}(t)$ at

2) For a rational function $f$ of $V, \operatorname{div}(f)$ denotes the divisor of $f$. 
$t=0$ [6, Theorem 1.1]. Hence $k^{\prime}=\overline{\boldsymbol{F}}_{p}(t)$ because every intermediate field $\left(\neq \bar{F}_{p}(t)\right)$ of $k / \bar{F}_{p}(t)$ is ramified at $t=0$. Thus $\widetilde{\text { End }} J_{\bar{F}_{p}(t)}=\operatorname{End}_{\bar{F}_{p}(t)} J_{\bar{F}_{p}(t)}$. Hence, in view of Proposition 3, Theorem 4 is reduced to (i) $f(X)$ is irreducible in $\overline{\boldsymbol{F}}_{p}(t)[X]$, and (ii) $g(X)=f(X) /(X-\theta)$ is irreducible in $\overline{\boldsymbol{F}}_{p}(t, \theta)[X]$, where $\theta(\in \bar{k})$ is a zero of $f(X)$. (i) follows from:

$$
\overline{\boldsymbol{F}}_{p}[X] \stackrel{\sim}{\longrightarrow} \overline{\boldsymbol{F}}_{p}[t, X] /(f(X)),
$$

and (ii) follows from:

$$
\overline{\boldsymbol{F}}_{p}[\theta, X] /\left(X^{2 g}+X^{2 g-1} \theta+\cdots+\theta^{2 g}+1\right) \stackrel{\sim}{\longrightarrow} \overline{\boldsymbol{F}}_{p}[t, \theta, X] /(g(X)),
$$

because $X^{2 g}+X^{2 g-1} \theta+\cdots+\theta^{2 g}+1$ is an irreducible polynomial in $\theta$ and $X$ if $p \nmid 2 g$.

q.e.d.

\section{References}

[1] P. Deligne and D. Mumford, The irreducibility of the space of curves of given genus, Inst. Hautes Études Sci. Publ. Math., No. 36, Paris, 1969.

[2] A. Grothendieck et al., Groupes de monodromie en géométrie algébrique (SGA 7 I), Springer Lecture Notes, No. 288.

[3] S. Mori, The endomorphism rings of some abelian varieties, Japan. J. Math., Vol. 1, 1975.

[4] D. Mumford, Abelian varieties, Oxford Univ. Press, London, 1970.

[5 ] L. Rédei, Algebra, Akademische Verlagsgesellschaft Geest \& Portig K.-G., Leipzig, 1959.

[6] K. A. Ribet, Endomorphisms of semi-stable abelian varieties over number fields, Ann. of Math., Vol. 101, No. 3 (1975).

[7] B. L. van der Waerden, Algebra I, 6 Auflage, Grund. Math. Wiss., Bd. 33, Springer-Verlag, Berlin, 1964.

Department of Mathematics

KYOTO UNIVERSITY

Куото 606

JAPAN 